PROCEEDINGS OF THE

AMERICAN MATHEMATICAL SOCIETY

Volume 137, Number 2, February 2009, Pages 733-743

S 0002-9939(08)09502-6

Article electronically published on October 8, 2008

\title{
SETS OF CONSTANT DISTANCE FROM A COMPACT SET IN 2-MANIFOLDS WITH A GEODESIC METRIC
}

\author{
ALEXANDER BLOKH, MICHAŁ MISIUREWICZ, AND LEX OVERSTEEGEN
}

(Communicated by Alexander N. Dranishnikov)

\begin{abstract}
Let $(M, d)$ be a complete topological 2-manifold, possibly with boundary, with a geodesic metric $d$. Let $X \subset M$ be a compact set. We show then that for all but countably many $\varepsilon$ each component of the set $S(X, \varepsilon)$ of points $\varepsilon$-distant from $X$ is either a point, a simple closed curve disjoint from $\partial M$ or an $\operatorname{arc} A$ such that $A \cap \partial M$ consists of both endpoints of $A$ and that arcs and simple closed curves are dense in $S(X, \varepsilon)$. In particular, if the boundary $\partial M$ of $M$ is empty, then each component of the set $S(X, \varepsilon)$ is either a point or a simple closed curve and the simple closed curves are dense in $S(X, \varepsilon)$.
\end{abstract}

\section{INTRODUCTION}

The boundary of a Euclidean ball on the plane (or, equivalently, the set $S(x, \varepsilon)$ of points $\varepsilon$-distant from a given point $x$ ) is a simple closed curve. If instead of a point $x$ we consider a compact set $X \subset \mathbb{R}^{2}$, then the same claim about the set $S(X, \varepsilon)$ of all points $\varepsilon$-distant from $X$ is not true. Thus, it is natural to ask what are the topological types of the sets $S(X, \varepsilon)$ and whether, apart from a small set of distances $\varepsilon$, the set $S(X, \varepsilon)$ can be guaranteed to be a "topologically simple" set.

A strong result in this direction is due to M. Brown, who showed in [4] that given a compact subset $X$ of the plane, for all but countably many $\varepsilon>0$ the set $S(X, \varepsilon)$ is the union of pairwise disjoint points, arcs, and simple closed curves (see also [3] for related results). In this paper we generalize the results of 4 in two directions. Instead of the plane with Euclidean metric, we consider 2-manifolds (not necessarily compact, with or without boundary) with metrics that make them proper geodesic metric spaces (see definitions below). Also, we improve the conclusions of the main theorem of [4. Our main result is Theorem 1.1.

In what follows we slightly abuse terminology and call singletons (sets with one element) points. We will also speak of point-components and arc-components of a set, meaning components that are points and arcs respectively. By "countable" we mean "of cardinality $\aleph_{0}$ or less", so finite sets are also countable.

Received by the editors February 8, 2007, and, in revised form, January 3, 2008.

2000 Mathematics Subject Classification. Primary 54E35, 54F15.

Key words and phrases. Finitely Suslinian, set of points of constant distance, geodesic space.

The first author was partially supported by NSF grant DMS 0456748.

The second author was partially supported by NSF grant DMS 0456526 .

The third author was partially supported by NSF grant DMS 0405774 . 
Theorem 1.1. Let a 2-manifold $M$ with metric d be a proper geodesic metric space. Let $X \subset M$ be compact. Then for all but countably many $\varepsilon>0$ each component of $S(X, \varepsilon)$ is either a point, a simple closed curve disjoint from the boundary $\partial M$ of $M$ or an arc $A$ such that $A \cap \partial M$ consists of both endpoints of $A$, and the union of arcs and simple closed curves is dense in $S(X, \varepsilon)$.

Observe that if $M$ has no boundary (e.g., if $M=\mathbb{R}^{2}$ ), then there are no arcs with endpoints in $\partial M$. Thus, we obtain the following corollary.

Corollary 1.2. Let a 2-manifold $M$ without boundary with metric $d$ be a proper geodesic metric space. Let $X \subset M$ be compact. Then for all but countably many $\varepsilon>0$ each component of $S(X, \varepsilon)$ is either a point or a simple closed curve, and the union of simple closed curves is dense in $S(X, \varepsilon)$.

Additional properties of the set $S(X, \varepsilon)$ can be found in Section 5 . We also prove in Theorem 2.2 that $S(\varepsilon)$ is finitely Suslinian for any $\varepsilon$ (planar finitely Suslinian sets are studied, e.g., in [1]). An example in Section [6] shows that the assumption that the metric is geodesic cannot be dropped.

Let us fix the terminology. We use some standard notions, e.g., arc, (2-) manifold (with or without boundary) and the boundary of a (2-)manifold. Note that if $M$ is a 2-manifold with boundary, then $\partial M$ is a 1-manifold without boundary. Let $(Y, d)$ be a metric space such that for every pair of points $x, y$ there exists an arc $I[x, y]$ with endpoints $x, y$ which is an isometric image of the interval $[0, d(x, y)]$ under a map $\psi:[0, d(x, y)] \rightarrow Y$ with $\psi(0)=x$ and $\psi(d(x, y))=y$. Then the metric $d$ is called a geodesic metric, $(Y, d)$ is called a geodesic space, and each arc $I[x, y]$ is called a geodesic segment between $x$ and $y$. There are many examples of geodesic 2-manifolds: smooth 2-manifolds without boundary with Riemannian metrics (if one adds a boundary, the distance between two points can be defined as the length of the shortest curve joining them), the plane with any norm and its convex closed subsets with nonempty interior, etc. (see [2]). A metric space is said to be proper if every closed ball is compact. Clearly, if $M$ is proper and $X$ is compact, then $S(X, \varepsilon)$ (and all components of $S(X, \varepsilon)$ ) are compact too. By the Hopf-Rinow Theorem (see, e.g., [2]), a locally compact geodesic space is proper if and only if it is complete. From now on we assume that $Y=M$ is a 2-manifold and fix a metric $d$ on $M$ which is compatible with the standard topology on $M$ and makes $(M, d)$ a proper geodesic space.

In what follows all standard notions (a circle, a ball, etc.) are understood in the sense of the metric $d$. Denote by $B(x, \varepsilon)$ the ball of radius $\varepsilon$ centered at $x$. Then $\partial B(x, \varepsilon)$ coincides with the set $S(x, \varepsilon)=\{z \in M: d(z, x)=\varepsilon\}$. Let $X$ be a compact subset of $M$; from now on we fix the set $X$ and often omit referring to $X$ (thus, we use the notation $S(\varepsilon)$ rather than $S(X, \varepsilon)$, etc.). Let $d(x)=\inf \{d(x, y): y \in X\}$; i.e., $d(x)$ is the distance from a point $x$ to the set $X$. Since $X$ is compact, there exists a point $z \in X$ with $d(x)=d(x, z)$ and hence there exists a geodesic segment $I[x, z]$. Any such segment will be called an $x$-twig and will be denoted $I[x]$. A twig $I[x]$ is disjoint from $S(d(x))$ except for its endpoint $x$; this is widely used in the following.

Suppose that $I \subset M$ is an arc in $M$ such that no point of $I$, except perhaps endpoints, belong to $\partial M$. Then if none of the endpoints of $I$ is in $\partial M$ (that is, the whole arc is disjoint from $\partial M$ ), we will call $I$ loose. If one endpoint is in $\partial M$ and 
the other is not there, we will call $I$ semi-loose. If both endpoints are in $\partial M$, we will call $I$ fastened. Thus, the arcs mentioned in Theorem 1.1 are fastened arcs.

The authors would like to thank the referee for useful remarks.

\section{ONLY POINTS, ARCS AND SIMPLE CLOSED CURVES}

In this section we extend Brown's results 4 to the situation described above. For given two disjoint closed sets $A, B \subset M$, a continuum $C$ is said to be irreducible between $A$ and $B$ if $C$ intersects both $A$ and $B$ and does not contain a subcontinuum with the same property. Given a continuum $D$ intersecting $A$ and $B$, one can use Zorn's Lemma to find a subcontinuum $C \subset D$ irreducible between $A$ and $B$.

Lemma 2.1. Let $K$ be an irreducible continuum between $\partial U$ and $\partial V$ where $U, V$ are open sets with disjoint closures. Then $K$ is disjoint from both $U$ and $V$.

Proof. Set $K^{\prime}=K \backslash \bar{V}$. Take a component $Y$ of $\overline{K^{\prime}}$ containing a point from $\partial U$. By the Boundary Bumping Theorem (Theorem 5.4 from [7, Chapter V, p. 73]) $Y$ intersects $\partial V$. Since $K$ is irreducible, $Y=K$ and hence $K$ is disjoint from $V$. Similarly, $K$ is disjoint from $U$.

Below convergence of continua is understood in the Hausdorff sense. A compactum $Z$ is said to be finitely Suslinian if for each $\varepsilon>0$, each collection of pairwise disjoint subcontinua of diameter larger than $\varepsilon$ is finite. It is known that each finitely Suslinian continuum is locally connected and arcwise connected 7 .

Theorem 2.2. The set $S(\varepsilon)$ is finitely Suslinian.

Proof. By the assumptions, $S(\varepsilon)$ is compact. Suppose it is not finitely Suslinian. Then there exists a sequence of pairwise disjoint continua $K_{i} \subset S(\varepsilon)$ with diameters bounded away from zero. We may assume that they converge to a continuum $K \subset S(\varepsilon)$. Then $K$ is disjoint from $X$. By taking a smaller subcontinuum, we may assume that there exists a neighborhood $W$ of $K$ such that $\bar{W} \cap X=\emptyset$ and $\bar{W}$ is homeomorphic to a closed disk. Choose points $x \neq y \in K$ and set $\rho=d(x, y)$. Choose small connected neighborhoods $U \subset W$ of $x$ and $V \subset W$ of $y$ so that $\bar{U}$ and $\bar{V}$ are both homeomorphic to a closed disk, and $U \subset B(x, \rho / 8)$ and $V \subset B(y, \rho / 8)$ (see Figure 11). Any continuum $K_{i}$ with big enough $i$ intersects both $\partial U$ and $\partial V$. For every $i$ choose a continuum $K_{i}^{\prime} \subset K_{i}$ irreducible between $\partial U$ and $\partial V$. We may assume that $K_{i}^{\prime}$ 's converge to a continuum $K^{\prime \prime} \subset K$. By Lemma 2.1 all sets $K_{i}^{\prime}$

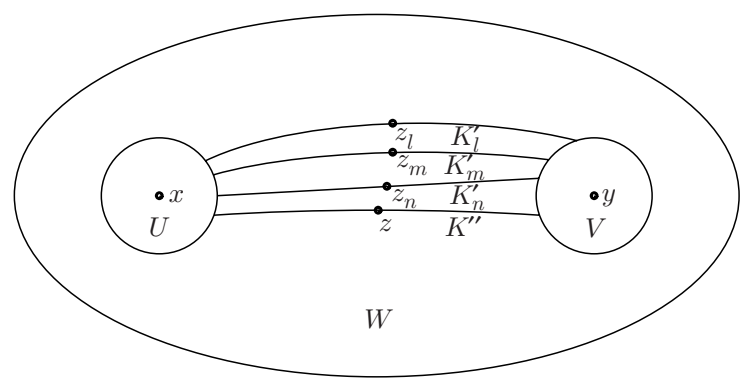

Figure 1. Construction from the proof of Theorem 2.2 
and hence the set $K^{\prime \prime}$ are disjoint from $U \cup V$, and we may assume that they are all contained in $W$.

For points $t \in \partial U \cap K^{\prime \prime}$ we have $d(x, t) \leq \rho / 8$, while for points $t \in \partial V \cap K^{\prime \prime}$ by the triangle inequality we have $d(x, t) \geq 7 \rho / 8$. Hence, since $K^{\prime \prime}$ is connected, there is a point $z \in K^{\prime \prime}$ with $d(z, x) \geq \rho / 2, d(z, y) \geq \rho / 2$. Since $K_{i}^{\prime}$ converge to $K^{\prime \prime}$, there exists a sequence of points $z_{i} \in K_{i}^{\prime}, z_{i} \rightarrow z$. We may assume that $d\left(z_{i}, z\right)<\rho / 8$ for all $i$. For each $z_{i}$ there exists a $z_{i}$-twig $I_{i}=I\left[z_{i}, x_{i}\right]$ with $x_{i} \in X$ and $d\left(z_{i}, x_{i}\right)=\varepsilon$. Then $I_{i} \backslash\left\{z_{i}\right\}$ is disjoint from $S(\varepsilon)$ and hence from all the sets $K_{r}^{\prime}$ and the set $K^{\prime \prime}$. We show that for metric reasons $I_{i} \backslash\left\{z_{i}\right\}$ is disjoint from $U$ or $V$, while for topological reasons it is trapped between various sets $K_{r}^{\prime}$. Since to reach out to $X$ the twig $I_{i}$ has to exit $W$, this leads to a contradiction.

To implement this plan let us first show that $I_{i}$ is disjoint from both $\bar{U}$ and $\bar{V}$. Suppose otherwise and choose, say, $t \in I_{i} \cap \bar{V}$. Since $d(z, y) \geq \rho / 2, d\left(z_{i}, z\right)<\rho / 8$ and $d(t, y) \leq \rho / 8$, by the triangle inequality $d\left(z_{i}, t\right) \geq \rho / 2-\rho / 8-\rho / 8=\rho / 4$. Thus, $d\left(t, x_{i}\right) \leq \varepsilon-\rho / 4$. On the other hand, $d(t, y) \leq \rho / 8$ and hence $d\left(y, x_{i}\right) \leq$ $\varepsilon-\rho / 4+\rho / 8=\varepsilon-\rho / 8$, a contradiction with $y \in S(\varepsilon)$.

To implement the second part choose three distinct continua $K_{l}^{\prime}, K_{m}^{\prime}$ and $K_{n}^{\prime}$ and then consider twigs $I_{l}, I_{m}$ and $I_{n}$. Clearly, $d\left(I_{l} \cup K_{l}^{\prime}, K_{m}^{\prime} \cup K_{n}^{\prime}\right)>0, d\left(I_{m} \cup K_{m}^{\prime}\right.$, $\left.K_{l}^{\prime} \cup K_{n}^{\prime}\right)>0$ and $d\left(I_{n} \cup K_{n}^{\prime}, K_{l}^{\prime} \cup K_{m}^{\prime}\right)>0$. Choose $\gamma>0$ less than one-tenth of the minimal of these three distances. Now, let $J_{l}$ be an arc with endpoints on $\partial U$ and $\partial V$, otherwise disjoint from $\partial U \cup \partial V$, which contains $z_{l}$ and is $\gamma$-close to $K_{l}^{\prime}$. Define arcs $J_{m}, J_{n}$ similarly. Collapse $\bar{U}$ and $\bar{V}$ to two points. Then by Kuratowski's " $\theta$-curve theorem" (Theorem 2 from [5, vol. 2, Chapter 10, §61, II, p. 511]) we may assume that the continuum $Z=J_{l} \cup J_{n} \cup \bar{U} \cup \bar{V}$ separates $z_{m}$ from $\partial W$. On the other hand, by the choice of $\gamma$ the twig $I_{m}$ connects $z_{m}$ and $X$ and bypasses $J_{l} \cup J_{n}$, a contradiction because $I_{m}$ must intersect $\partial W$ since $\bar{W} \cap X=\emptyset$.

Since by [6] there are only countably many pairwise disjoint triods in the plane, then for all but countably many values of $\varepsilon$ the set $S(\varepsilon)$ contains no triods (this argument is similar to 4]). A component of a finitely Suslinian compactum is a locally connected, and hence arcwise connected, continuum. Therefore every component of $S(\varepsilon)$ is either a point, an arc, or a simple closed curve.

Theorem 2.3. For all but countably many $\varepsilon>0$ each component of $S(\varepsilon)$ is a point, an arc, or a simple closed curve.

Denote by $T_{1} \subset \mathbb{R}^{+}=(0, \infty)$ the set of all parameters $\varepsilon$ which satisfy the conditions of Theorem 2.3. By this theorem, the set $\mathbb{R}^{+} \backslash T_{1}$ is countable.

\section{HOW COMPONENTS INTERSECT THE BOUNDARY}

In this short section we make the first improvement to Theorem 2.3. It applies only to manifolds with boundary and has no effect if there is no boundary. By an interior point of an arc $A$ we mean a point $x \in A$ which is not an endpoint of $A$.

Lemma 3.1. Any family of pairwise disjoint arcs in $M$, each of whom has an interior point in $\partial M$, is countable.

Proof. Consider the space $L$ in the product $M \times[0,1)$, which is the union of $M \times\{0\}$ and $\partial M \times[0,1)$. It is a 2-manifold without boundary. If $I$ is an arc in $M$ whose interior point $x$ belongs to $\partial M$, then the union of $I$ and $\{x\} \times[0,1 / 2]$ is a triod in 
$L$, and for disjoint $\operatorname{arcs} I, J$ those triods are disjoint. Therefore there can be only countably many such pairwise disjoint arcs.

Since components of $S\left(\varepsilon_{1}\right)$ and $S\left(\varepsilon_{2}\right)$ for $\varepsilon_{1} \neq \varepsilon_{2}$ are disjoint, we get the following theorem as a corollary to this lemma.

Theorem 3.2. For all but countably many $\varepsilon>0$ each arc-component of $S(\varepsilon)$ has no interior point in $\partial M$ and each component of $S(\varepsilon)$ which is a closed curve is disjoint from $\partial M$.

Let $T_{2}$ be the set of all $\varepsilon \in T_{1}$ which satisfy the conditions of Theorem 3.2. In the future consider only $\varepsilon \in T_{2}$ (thus, all arc-components of $S(\varepsilon)$ will be loose, semi-loose or fastened). Then by Theorem 3.2 the set $\mathbb{R}^{+} \backslash T_{2}$ is countable.

\section{Two SIDES OF A LOCAL ARC}

In this section we consider local properties of the function $d$ near components of $S(\varepsilon)$ which are not point-components. Together with components of $S(\varepsilon)$ we will consider components of its complement and sometimes components of other sets. To minimize confusion, we will think of $M$ with $S(\varepsilon)$ as a map (in a geographical sense) that we try to color with two colors. Thus, we will refer to the components of $M \backslash S(\varepsilon)$ as countries. A country will be called white if its intersection with $X$ is non-empty and black otherwise.

Lemma 4.1. On each country the sign of the function $d(\cdot)-\varepsilon$ is constant. It is positive on black countries and negative on white ones.

Proof. The function $d(\cdot)-\varepsilon$ on a country is a non-zero continuous function on a connected set, and therefore its sign is constant. If this sign is negative, then each point of the country has a twig contained in the country, so there are points of $X$ there. Therefore the country is white. If the sign is positive, then there cannot be points of $X$ there, because for them $d$ is zero. Therefore the country is black.

Now we prove an important technical lemma. By an open Jordan disk we mean a set $U$ homeomorphic to the open unit disk, whose boundary is homeomorphic to the circle. Denote the diameter of a set $E$ by $\operatorname{diam}(E)$. Also, let $K(M)$ be the space of all compact subsets of $M$ with the Hausdorff metric. Then $K(M)$ has a countable base. Let $\mathcal{A}$ be the subspace of $K(M)$ consisting of components of all sets $S(\xi), \xi>0$; then $\mathcal{A}$ has a countable base too. The function $\psi: \mathcal{A} \rightarrow \mathbb{R}^{+}$, given by $\psi(Z)=\xi$ if $Z$ is a component of $S(\xi)$, is continuous.

Lemma 4.2. Let $C \subset M$ be a simple closed curve, bounding an open Jordan disk $U$ disjoint from $X$. Let $B$ be a component of $S(\varepsilon)$ and let $A$ be a component of $B \cap U$ such that $\bar{A}$ is an arc.

(1) Suppose that at least one endpoint of $A$ belongs to $C$ and $Q$ is a component of $U \backslash A$.

(a) Assume that there is a country $W$ and a sequence $x_{n} \in W \cap Q$ converging to $x \in A$. If $y_{n} \in Q \backslash S(\varepsilon), n=1,2, \ldots$, is a sequence converging to $y \in A$ (we do not exclude $x=y$ ), then for all sufficiently large $n$ the points $y_{n}$ belong either to $W$ or to countries contained in $Q$ and bounded by simple closed curves.

(b) There is only one country $W$ such that the closure of $W \cap Q$ intersects A. 


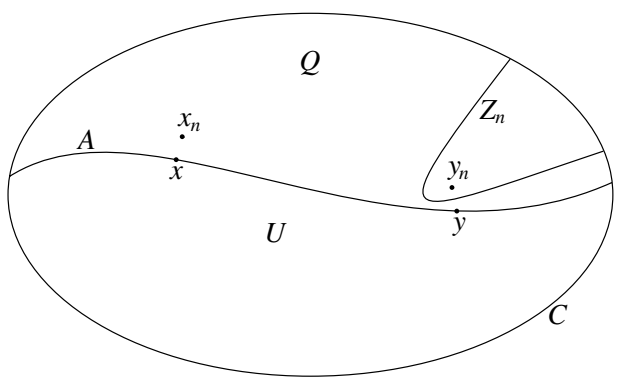

Figure 2. Construction from the proof of Lemma 4.2 (1)

(2) Suppose that both endpoints of $A$ belong to $C$. Let $Q, R$ be the components of $U \backslash A$, and let $W, V$ be white countries such that $\overline{W \cap Q}, \overline{V \cap R}$ intersect $A$. Then $\psi$ attains a strict local maximum at $B$.

Proof. (1) It follows from the assumptions that $Q$ is homeomorphic to an open disk.

(a) If $y_{n} \notin W$, the set $S(\varepsilon) \cap Q$ separates $x_{n}$ from $y_{n}$ in $Q$. By a theorem of Kuratowski ([5. Theorem 1, p. 438]), there is a component $Z_{n}$ of $S(\varepsilon) \cap Q$ separating $x_{n}$ from $y_{n}$ in $Q$. If $\overline{Z_{n}}$ is a simple closed curve, then it bounds an open disk $V$. Since $U \cap X=\emptyset$, there are no points of $S(\varepsilon)$ in $V$ (otherwise their twigs would lead to points of $X$ in $V$ ), so $V$ is a country. If $\overline{Z_{n}}$ is not a simple closed curve, then it is an arc with both endpoints in $C$ (see Figure 2). Since $Z_{n}$ separates $y_{n}$ (or $x_{n}$ ) from $A$, for a large $n$ the geodesic segment $I\left[y_{n}, y\right]$ (or $I\left[x_{n}, x\right]$ ) intersects $Z_{n}$ at a point $z_{n}$, and $d\left(z_{n}, y\right)<d\left(y_{n}, y\right)$ (or $\left.d\left(z_{n}, x\right)<d\left(x_{n}, x\right)\right)$. Since $y_{n} \rightarrow y$ $\left(x_{n} \rightarrow x\right)$ and $\operatorname{diam}\left(Z_{n}\right) \geq d\left(z_{n}, \partial U\right), \operatorname{diam}\left(Z_{n}\right)>\min \{d(x, \partial U), d(y, \partial U)\} / 2$ for a large $n$. Thus, if there are infinitely many $n$ 's such that $y_{n}$ does not belong to $W$ or to a country contained in $Q$ and bounded by a simple closed curve, then there are infinitely many distinct (and therefore pairwise disjoint) sets $Z_{n}$ with $\operatorname{diam}\left(Z_{n}\right)>$ $\min \{d(x, \partial U), d(y, \partial U)\} / 2$, a contradiction, since $S(\varepsilon)$ is finitely Suslinian. This proves (a).

(b) Clearly, there is a country $W$ such that the closure of $W \cap Q$ intersects $A$. If there is another such country $V$, then by (a) it must be contained in $Q$ and bounded by a simple closed curve. However, then $\bar{V}$ cannot intersect $A$, a contradiction.

(2) Suppose that $\psi$ does not attain a strict local maximum at $B$. Then there is a sequence $Y_{n}, n=1,2 \ldots$, of components of $S\left(\xi_{n}\right)$ respectively, convergent in the Hausdorff metric to $B$ and such that $\xi_{n} \geq \varepsilon$ for each $n$. Since $S(\varepsilon)$ is finitely Suslinian, only finitely many $\xi_{n}$ can be equal to $\varepsilon$, so we can assume that $\xi_{n}>\varepsilon$ for all $n$. We can choose a sequence of points $y_{n} \in Y_{n}$ convergent to some $y \in A$, and we can assume that all $y_{n}$ belong to $Q$. Since $W$ is white, by Lemma 4.1 those points do not belong to $W$. Since $\overline{W \cap Q}$ intersects $A$, there exists a sequence of points $x_{n} \in W \cap Q$ convergent to some $x \in A$. Therefore, by (1)(a), for sufficiently large $n$ the point $y_{n}$ belongs to a country $W_{n}$ contained in $Q$ and bounded by a simple closed curve. Then $Y_{n} \subset W_{n}$, which contradicts the assumption that $Y_{n}$ converge in the Hausdorff metric to $B$. This completes the proof.

Remark 4.3. In the setup of Lemma 4.2 (2) $W$ and $V$ cannot both be black because the twigs of the points of $A$ cannot pass through a black country. 
Now we can eliminate loose ends.

Lemma 4.4. Let $B$ be a loose or semi-loose arc-component of $S(\varepsilon)$. Then $\psi$ attains a strict local maximum at $B$.

Proof. Let $z$ be an endpoint of $B$ not contained in $\partial M$. There exists a neighborhood $U^{\prime}$ of $z$ which is an open Jordan disk disjoint from $X$, bounded by a simple closed curve $C^{\prime}$ and such that the closure of the component $A$ of $B \cap U^{\prime}$ containing $z$ is an arc with one of its endpoints in $C^{\prime}$. Then $Q^{\prime}=U^{\prime} \backslash A$ is connected. Therefore by Lemma 4.2 (1)(b) there is a unique country $W^{\prime}$ such that the closure of $W^{\prime} \cap Q^{\prime}$ intersects $A$. Hence, the twigs of points of $A$ (except their endpoints from $A$ ) are contained in $W^{\prime}$, so $W^{\prime}$ is white.

Take a point $t \in A$ different than $z$. Then we can find a neighborhood $U$ of $t$ as in Lemma 4.2 (2) and contained in $U^{\prime}$. Then both countries $W, V$ from Lemma 4.2 (2) are equal to $W^{\prime}$ (so they are white), and hence by that lemma $\psi$ attains a strict local maximum at $B$.

Since the space $\mathcal{A}$ is separable, the set of points at which $\psi$ attains a strict local maximum is countable. Therefore the set of values $\varepsilon$ at such points is countable. Denote by $T_{3}$ the set of all parameters $\varepsilon \in T_{2}$ which are not strict local maximum values of $\psi$. Then the set $\mathbb{R}^{+} \backslash T_{3}$ is countable. From now on, we will consider only $\varepsilon \in T_{3}$. Thus, all arc-components of $S(\varepsilon)$ will be fastened. Moreover, in the situation described in Lemma 4.2 (2) $, W, V$ cannot both be white. They also cannot both be black by Remark4.3. Now we make the last step towards the proof of Theorem 1.1.

Lemma 4.5. Assume that a point-component $x$ of $S(\varepsilon)$ does not belong to the closure of $\operatorname{Sep}(\varepsilon)$. Then d attains a local maximum at $x$.

Proof. Suppose that $d$ does not attain a local maximum at $x$. Then there is a sequence of points $x_{n} \rightarrow x$ such that $d\left(x_{n}\right)>\varepsilon$. By Lemma 4.1, each $x_{n}$ belongs to a black component of $M \backslash S(\varepsilon)$. Therefore a geodesic segment joining $x$ with $x_{n}$ intersects the boundary of this component, so it intersects $\operatorname{Sep}(\varepsilon)$. The points of intersection converge to $x$ as $n \rightarrow \infty$.

Since the space $M$ is separable, the set of values of $\varepsilon$ at which $d$ attains a local maximum is countable. Denote by $T_{4}$ the set of all parameters $\varepsilon \in T_{3}$ which are not local maximum values of $d$. Then the set $\mathbb{R}^{+} \backslash T_{4}$ is countable, and the proof of Theorem 1.1 is complete.

\section{MAP COLORING}

In this section we obtain additional information about the way that $S(\varepsilon)$ (for $\varepsilon \in T_{4}$ ) divides $M$. We already know by Lemma 4.2 and Remark 4.3 that if two countries share a piece of border, they are opposite colors. However, our map may have infinitely many countries, which does not allow us to get a clear picture.

Call a component $Z$ of $S(\varepsilon)$ which is not a point bounding or non-bounding, according to whether (together with a piece of $\partial M$ if $Z$ is an arc) it bounds a Jordan disk. If $M$ is not homeomorphic to the 2 -sphere or the closed unit disk, for each bounding component $Z$ of $S(\varepsilon)$ there is a unique disk $D(Z)$ bounded by it. If $M$ is homeomorphic to the 2 -sphere, we fix such a homeomorphism. If $Z$ is contained is a hemisphere, we take as $D(Z)$ the disk contained in this hemisphere. For the 
remaining finitely many bounding components $Z$, we choose a point $p \notin S(\varepsilon)$ and denote by $D(Z)$ the disk bounded by $Z$ such that $p \notin D(Z)$. If $M$ is homeomorphic to the closed unit disk, the problem occurs only if $Z$ is a fastened arc. Then we make a similar construction as for the 2 -sphere. In the disk model, we choose the component that is contained in a half-disk, and for the remaining finitely many fastened arcs we fix a point $p \notin S(\varepsilon)$ and choose as $D(Z)$ the disk bounded by $Z$ and a part of the boundary of the disk that does not contain $p$. Call a bounding component special if $D(Z) \cap X \neq \emptyset$ and general otherwise.

Lemma 5.1. There are only finitely many non-bounding and special bounding components of $S(\varepsilon)$.

Proof. Since the set $S(\varepsilon)$ is compact, it can be covered by finitely many open sets homeomorphic to a disk or a half-disk. This cover has a Lebesgue number. Therefore there exists $\delta>0$ such that any component of $S(\varepsilon)$ of diameter less than $\delta$ is bounding. Since $S(\varepsilon)$ is finitely Suslinian, it follows that there are only finitely many non-bounding components of $S(\varepsilon)$.

It is easy to see that if we take a sufficiently small $\eta>0$, then for any bounding component $Z$ of diameter less than $\eta$ the diameter of $D(Z)$ is smaller than $\varepsilon$. If $Z$ is special, then $\operatorname{diam}(D(Z)) \geq \varepsilon$, so $\operatorname{diam}(Z) \geq \eta$. Therefore there are only finitely many special bounding components.

Consider non-bounding and special bounding components of $S(\varepsilon)$; this gives us a new map with finitely many countries. Since for a general bounding component $Z$ the set $D(Z)$ contains no points of $S(\varepsilon)$ (otherwise twigs of those points would lead to points of $X$ in $D(Z))$, such $D(Z)$ is a black country. We will call such a country a black dot.

Theorem 5.2. Consider the map $\mathcal{M}$ on $M$ given by the non-bounding and special bounding components of $S(\varepsilon)$ as boundaries. It can be colored by two colors in such a way that no two countries with the same color are neighbors.

Proof. By Lemma 4.2 and Remark 4.3, across the border of a black dot there is a white country. Thus, if we repaint each black dot (together with its boundary) white, we will diminish the number of countries, but each new country will have a unique color, and the property that two countries with a common piece of border have opposite colors will be preserved. The map we get in this way is $\mathcal{M}$.

It is clear that the countries that are non-compact are black. Therefore from Theorem 5.2 we immediately get the following corollary.

Corollary 5.3. For every component of $\partial M$ the number of endpoints of nonbounding fastened arcs belonging to this component is even.

\section{EXAMPLE}

In this section we show that our main theorem (and the corollary to it) may not hold if we drop the assumption that the metric is geodesic.

We need a real function with some special properties. While any function with a fractal graph should have those properties, we define one for which the proof is especially simple. 

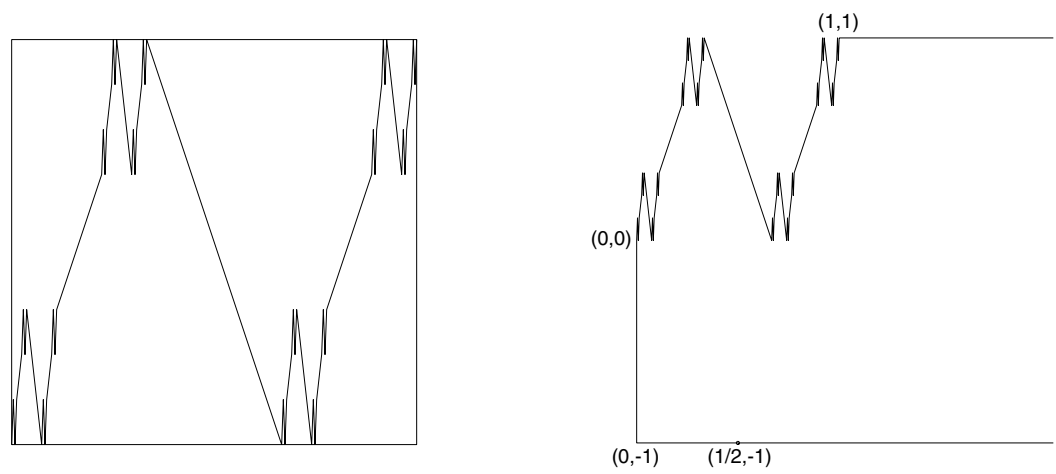

Figure 3 . The graph of $f$ and the curve $L$

Lemma 6.1. There exist a continuous function $f:[0,1] \rightarrow[0,1]$ and an uncountable set $C \subset[0,1]$ such that for every $x \in C$ there is an uncountable set $E(x) \subset f^{-1}(x)$ such that for every $y \in E(x)$ and every $\delta>0$ there exist points $y_{-}, y_{+} \in[0,1]$ with $y-\delta<y_{-}<y<y_{+}<y+\delta$ and $f\left(y_{-}\right), f\left(y_{+}\right)>f(y)=x$.

Proof. Let $K$ be the standard middle-thirds Cantor set. In other words, $K$ consists of points that have ternary expansion $0 . x_{1} x_{2} x_{3} x_{4} \ldots$ with $x_{i} \in\{0,2\}$ for all $i$. For such $x$ define $f(x)$ as the number with ternary expansion $0 . x_{2} x_{4} x_{6} x_{8} \ldots$ Clearly, this function is continuous and therefore can be extended to a continuous function $f:[0,1] \rightarrow[0,1]$, for instance linearly on each gap in $K$ (see Figure 3). Let $C$ be the set of points of $K$ which are not endpoints of the gaps in $K$, so in their ternary expansions both 0 and 2 appear infinitely many times. The set $C$ is uncountable. Fix $x \in C$ with the ternary expansion $0 . x_{1} x_{2} x_{3} x_{4} \ldots$ Let $C(x)$ be the set of all numbers with the ternary expansion $0 . y_{1} x_{1} y_{2} x_{2} \ldots$, where $y_{i} \in\{0,2\}$ for all $i$ and both 0 and 2 appear infinitely many times among $y_{i}$. Clearly, $C(x) \subset f^{-1}(x)$. For a given $y \in C(x)$ (with the ternary expansion $0 . y_{1} x_{1} y_{2} x_{2} \ldots$ ) and $\delta>0$, in order to get $y_{-}$(respectively $y_{+}$) with the properties described in the statement of the lemma, find a sufficiently large $n$ such that $y_{n}=2$ (respectively $y_{n}=0$ ) and $m>n$ such that $x_{m}=0$ and replace $y_{n}$ by 0 (respectively by 2 ) and $x_{m}$ by 2 in the ternary expansion of $y$.

Now set $G=\{(x, f(x)): x \in[0,1]\}$ and define the curve $L$ in $\mathbb{R}^{2}$ :

$$
L=G \cup\{(x, 1): x \in[1, \infty)\} \cup\{(0, y): y \in[-1,0]\} \cup\{(x,-1): x \in[0, \infty)\}
$$

(see Figure 3). Then the set $M=L \times \mathbb{R} \subset \mathbb{R}^{3}$ is homeomorphic to the plane $\mathbb{R}^{2}$. We use in $\mathbb{R}^{3}$ the max norm and the metric $d$ induced by it, so

$$
d\left((x, y, z),\left(x^{\prime}, y^{\prime}, z^{\prime}\right)\right)=\max \left(\left|x-x^{\prime}\right|,\left|y-y^{\prime}\right|,\left|z-z^{\prime}\right|\right) .
$$

With this metric restricted to $M$ (we will denote it also by $d$ ), $M$ is a complete 2-manifold without boundary. However, $d$ is not a geodesic metric on $M$ (although it is on $\mathbb{R}^{3}$ ).

Let us set $X=\{(1 / 2,-1,0)\}$ (the point $(1 / 2,-1)$ is marked in Figure 3). Then the set $S(\varepsilon)$ will be the intersection of $M$ with the sphere centered at $(1 / 2,-1,0)$ of radius $\varepsilon$ in $\left(\mathbb{R}^{3}, d\right)$. This sphere is geometrically the cube centered at $(1 / 2,-1,0)$ 
with edges parallel to the coordinate axes and of length $2 \varepsilon$. To visualize the whole picture, imagine the $x$-axis being horizontal and pointing to the right, the $y$-axis vertical pointing up, and the $z$-axis horizontal pointing towards the visualizer.

Lemma 6.2. For every $c \in C$ and $b \in E(c)$ the segment

$$
A(c, b)=\{(x, y, z): x=b, y=c, z \in[-1-c, 1+c]\}
$$

is a component of $S(1+c)$.

Proof. Fix $c \in C$ and $b \in E(c)$ and denote $A=A(c, b)$. Clearly, $A \subset S(1+c)$. By Lemma 6.1, for every $\delta>0$ there exist points $b_{-}, b_{+} \in[0,1]$ with $b-\delta<b_{-}<b<$ $b_{+}<b+\delta$ and $f\left(b_{-}\right), f\left(b_{+}\right)>f(b)=c$. Let $c^{\prime}=\min \left(f\left(b_{-}\right), f\left(b_{+}\right)\right)$and set

$$
a_{-}=\sup \left\{x<b: f(b)=c^{\prime}\right\}, \quad a_{+}=\inf \left\{x>b: f(b)=c^{\prime}\right\} .
$$

Then $b-\delta<a_{-}<b<a_{+}<b+\delta, f\left(a_{-}\right)=f\left(a_{+}\right)=c^{\prime}>f(b)$ and $f(x)<c^{\prime}$ for every $x \in\left(a_{-}, a_{+}\right)$. The two segments

$$
\left\{(x, y, z): x=a_{ \pm}, y=c^{\prime}, z \in\left[-1-c^{\prime}, 1+c^{\prime}\right]\right\}
$$

are contained in $S\left(1+c^{\prime}\right)$, and so are the two arcs

$$
\left\{(x, y, z): x \in\left[a_{-}, a_{+}\right], y=f(x), z= \pm\left(1+c^{\prime}\right)\right\} \text {. }
$$

Their union is a simple closed curve whose projection to the $x z$-plane (the horizontal one) is the boundary of the rectangle $R(\delta)$ with the vertices at the points $\left(a_{ \pm}, \pm(1+\right.$ $\left.c^{\prime}\right)$ ). The projection $\pi(A)$ of $A$ is the segment with the endpoints $(b, \pm(1+c))$. Thus, $\pi(A)$ is contained in $R(\delta)$, and since the sets $S(1+c)$ and $S\left(1+c^{\prime}\right)$ are disjoint, the projection of the component of $S(1+c)$ containing $A$ is contained in $R(\delta)$. Moreover, the intersection of the rectangles $R(\delta)$ over all $\delta>0$ is equal to $\pi(A)$. Taking into account that this projection on the set $\{(x, f(x)): x \in[0,1]\} \times \mathbb{R}$ is a homeomorphism onto the image, this proves that $A$ is a component of $S(1+c)$.

As an immediate consequence of Lemmas 6.1 and 6.2 we get the following theorem, which is the main result of this section.

Theorem 6.3. There exists a 2-manifold $M$ with a complete metric $d$, homeomorphic to $\mathbb{R}^{2}$, such that for an uncountable set of values of $\varepsilon$ the set $S(\varepsilon)$ has uncountably many arc-components.

\section{REFERENCES}

1. A. Blokh, M. Misiurewicz and L. Oversteegen, Planar finitely Suslinian compacta, Proc. Amer. Math. Soc. 135 (2007), 3755-3764. MR.2336592

2. M. R. Bridson and A. Haefliger, Metric Spaces of Non-positive Curvature, Springer-Verlag, Berlin, 1999. MR 1744486 (2000k:53038)

3. G. A. Brouwer, Green's functions from a metric point of view, Ph.D. dissertation, University of Alabama at Birmingham, 2005.

4. M. Brown, Sets of constant distance from a planar set, Michigan Math. J. 19 (1972), 321-323. MR 0315714 (47:4263)

5. K. Kuratowski, Topology II, Academic Press, New York, 1968. MR0259835(41:4467) 
6. R. L. Moore, Concerning triods in the plane and junction points of plane continua, Proc. Nat. Acad. Sci. U.S.A. 14 (1928), 85-88.

7. S. B. Nadler, Jr., Continuum theory. An introduction, Monographs and Textbooks in Pure and Applied Mathematics 158, Marcel Dekker, Inc., New York, 1992. MR.1192552 (93m:54002)

Department of Mathematics, University of Alabama in Birmingham, University Station, Birmingham, Alabama 35294-2060

E-mail address: ablokh@math.uab.edu

Department of Mathematical Sciences, IUPUi, 402 N. Blackford Street, IndianapoLIS, INDIANA 46202-3216

E-mail address: mmisiure@math.iupui.edu

Department of Mathematics, University of Alabama in Birmingham, University Station, Birmingham, Alabama 35294-2060

E-mail address: overstee@math.uab.edu 